\title{
Postextinction of Conditioned Fear: Between Two CS-Related Memories
}

\author{
René Garcia ${ }^{1}$ \\ Neurobiologie Comportementale, Université de Nice-Sophia Antipolis, Nice, France
}

In his classical studies, Pavlov (1927) demonstrated that when a neutral stimulus is frequently paired with an event (unconditioned stimulus: US), which always produces innate or unconditioned responses, it becomes capable (conditioned stimulus: CS) of eliciting learned or conditioned responses. Conditioned responses typically have adaptive functions. However, conditioning of certain states, such as fear, can also be nonproductive. Fortunately, both animals and humans can exhibit opposing central mechanisms that weaken the strength of conditioned responses in certain conditions. For example, if after conditioning (CS-US pairing), the CS is repeatedly presented without the US, the strength of the conditioned response diminishes progressively over time. According to Pavlov (1927), this process, which he termed extinction, occurs as the result of the activation of a central inhibitory state that prevents the expression of conditioned responses. He also demonstrated that the engagement of this inhibitory state declines with the passage of time, a phenomenon known as spontaneous recovery (Pavlov 1927). This observation is not only true in appetitive conditioning (Pavlov's studies; Bouton 1993; Brooks and Bouton 1993), but has also been validated in aversive conditioning (Brooks et al. 1999). In this issue of Learning \& Memory, Quirk (2002) investigates several questions pertaining to the persistence of extinction memory and the development of spontaneous recovery and presents results indicating that conditioning and extinction of cued fear conditioning are mediated by independent systems.

Studies on postextinction effects of CS, particularly the phenomenon of spontaneous recovery, have fundamentally contributed to the understanding of certain aspects of the nature of extinction. For example, these studies indicate that extinction does not erase memory of conditioning (see Fig. 1). Thus, conditioning and extinction result from the formation of distinct CS-related memories that are separately accessible after the extinction period (Falls et al. 1992; Bouton 1993; Rescorla 2001; Santini et al. 2001). However, it remains unclear whether the extinction memory trace progressively fades with the passage of time

${ }^{1}$ E-MAIL rene.garcia@unice.fr; FAX (33)-4-9207-6162. Article and publication are at http://www.learnmem.org/cgi/doi/ $10.1101 / \mathrm{lm} .56402$. or whether this memory persists despite spontaneous recovery.

To examine these issues, Quirk (2002) measured spontaneous recovery at various time points up to $14 \mathrm{~d}$. Rats were previously conditioned to express freezing response toward a tone following tone-footshock (CS-US) pairings and then exposed to the CS-alone presentations for extinction of the conditioned fear, which was characterized by a progressive reduction of conditioned freezing to negligible levels. Remarkably, when rats were reexposed to the CSalone, they started displaying, from days 1-2, low levels of fear return (spontaneous recovery), which increased as a function of retention interval reaching $100 \%$ of preextinction values by day 10. Data from control rats, submitted to conditioning but not to extinction, showed that the passage of time alone did not increase the expression of conditioned freezing. Thus, the observed progressive spontaneous recovery was likely due to reduced activation of extinction memory rather than to increased activation of conditioning memory. The finding opens up the possibility that extinction memory is a short-lasting phenomenon that fades completely when long delays elapse between extinction and testing. Quirk also examined this possibility by resubmitting rats to extinction at day 14 . Although all rats exhibit a full return of conditioned fear on day 14 , they significantly reextinguished this fear faster than they did during the initial extinction, suggesting savings from the initial learning training (initial postconditioning CS-alone presentations).

If, following extinction, both forms of memory (extinction and conditioning memories) are simultaneously accessible, which factors preferentially activate one or the other type of memory during further exposure to the CS? Environmental cues have been suggested to play a key role. Bouton (1993) has suggested that the passage of time may produce temporal context changes, which may, in turn, incur a failure to retrieve the extinction memory. In this condition the perception of the CS automatically activates the CS-US memory (signaling a possible occurrence of the US); the outcome being stronger conditioned responses. In Quirk's study, because conditioning and extinction took place in the same context, it may be possible that with longer retention intervals the CS preferentially activated the CS-US memory rather than the CS-no-US memory. However, if such conditioning (i.e., tone-footshock pairings)

LEARNING \& MEMORY 9:361-363 @ 2002 by Cold Spring Harbor Laboratory Press ISSN1072-0502/02 \$5.00

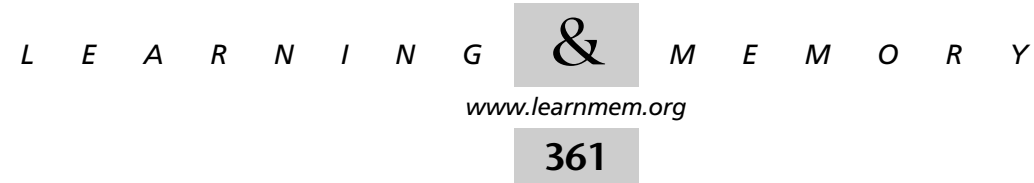




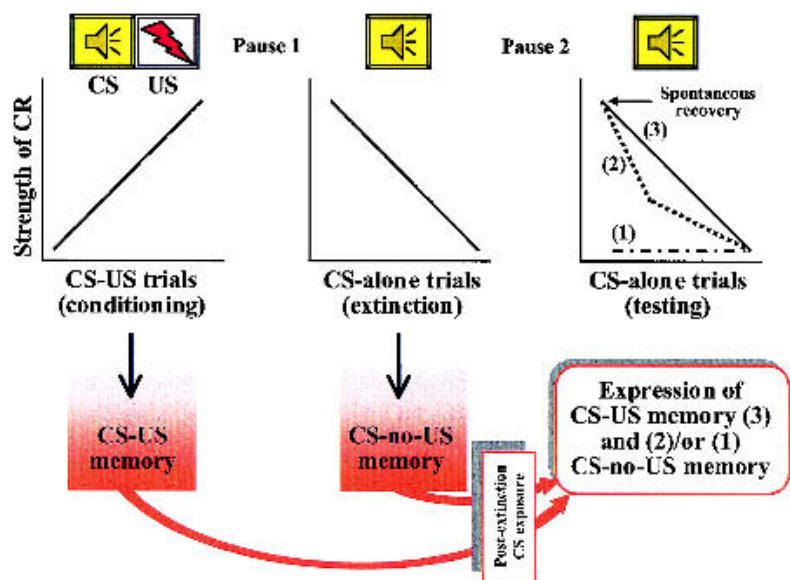

Figure 1 Changes in the strength of a conditioned response (CR). The strength of the CR (e.g., freezing behavior) increases (conditioning) over trials of CS-US (e.g., tone-footshock) pairing. Whatever the retention interval (Pause 1), CS-alone presentation produces high levels of the CR. Further presentations of the CS without the US results in a lowered strength of the CR (extinction). With the passage of time (Pause 2), the strength of the CR will either (1) remain low because of expression of CS-no-US memory; or (2) and (3) spontaneously recover because of expression of CS-US memory. In the latter case, additional CS-alone trials decline again the strength of the CR (2) with (Quirk 2002) or (3) without (Santini et al. 2001) savings from the initial extinction, according to the experimental conditions.

and extinction take place in different contexts, further presentations of the CS in the conditioning context preferentially activate the CS-US memory (Frohardt et al. 2000), a phenomenon known as renewal (Bouton and King 1983). In contrast, postextinction presentations of the CS in the extinction context can fully activate the CS-no-US memory even $7 \mathrm{~d}$ after the extinction training (Herry and Garcia 2002), demonstrating that mechanisms for long-term memory are involved in extinction of conditioned fear. Thus, as time passes after extinction, the CS-no-US memory remains available and can substantially interfere with CS-US memory retrieval in certain conditions.

Neither the brain structures nor the mechanisms implicated in postextinction memory selection, CS-US or CSno-US, are presently known. However, based on synaptic plasticity data, which is thought to underlie the acquisition and consolidation of memory, circuits in the medial prefrontal cortex and amygdala may be involved (Garcia 2002). First, retention of extinction is associated with an increase of synaptic efficacy in the medial prefrontal cortex, whereas spontaneous recovery is associated with decreased prefrontal synaptic efficacy (Herry and Garcia 2002). Second, the medial prefrontal cortex sends direct glutamatergic projections to the amygdala (McDonald et al. 1996) that inhibit transmission within the amygdala via activation of GABAergic interneurons (Rosenkranz and Grace 2002). Potentiation of glutamatergic transmission in the medial prefrontal cortex may, in turn, potentiate transmission between prefron- tal glutamatergic neurons and GABAergic interneurons in the amygdala through activation of $N$-methyl-D-aspartate (NMDA) receptors, a key mediator of synaptic plasticity. Third, microinjections of NMDA antagonists into the amygdala impair extinction of conditioned fear (Falls et al. 1992; Davis 2002). Likewise, microinjections of NMDA agonists facilitate extinction of conditioned fear (Davis 2002; Walker et al. 2002).

During CS reexposure, different levels of synaptic excitability between the prefrontal cortex and the amygdala may have different consequences on the behavior (see Fig. 2). Weak activation of this pathway (corresponding to a weak inhibition within the amygdala) promotes expression

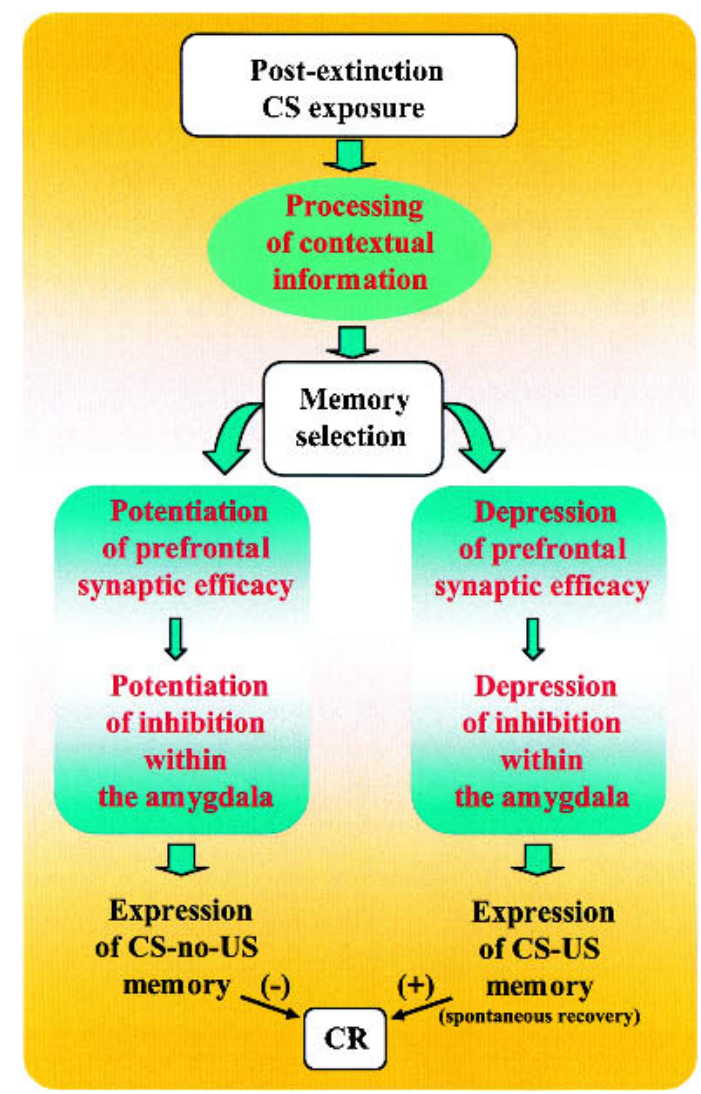

Figure 2 Postextinction of conditioned fear: memory selection. Because two types of CS-related memories (CS-no-US and CS-US) can be simultaneously accessible after the extinction period, reexposure to the CS (e.g., a tone initially paired with shock application) may yield memory selection or interactions between the two memories. Processing of contextual information may play a key role. For example, if a tone CS is presented in a context different from the conditioning environment, the tone-no-shock memory can be preferentially activated via potentiation of glutamatergic transmission between the medial prefrontal cortex and the amygdala, leading to inhibition (-) of CR (e.g., absence of freezing behavior). However, if CS reexposure takes place in the conditioning context (different from the extinction environment), the CS-US memory may be activated via depression of transmission between the medial prefrontal cortex and the amygdala, leading to spontaneous recovery $(+)$ of $\mathrm{CR}$.

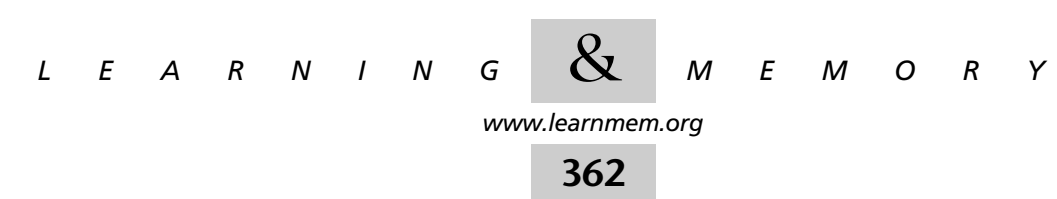


of CS-US memory, resulting in spontaneous recovery. In contrast, strong activation of this pathway facilitates the expression of CS-no-US memory, and consequently inhibits the spontaneous recovery phenomenon. Thus, this pathway may constitute part of the circuits involved in the regulation of the central inhibitory state suggested by Pavlov (1927).

The increased knowledge of the structure and functioning of these circuits will be of great interest both for clinical applications, because certain psychiatric disorders (e.g., posttraumatic stress disorder) derive from dysfunction in these circuits, and also for the basic understanding of memory processes.

\section{REFERENCES}

Bouton, M.E. 1993. Context, time, and memory retrieval in the interference paradigms of Pavlovian learning. Psychol. Bull. 114: 80-99.

Bouton, M.E. and King, D.A. 1983. Contextual control of the extinction of conditioned fear: Tests for the associative value of the context. J. Exp. Psychol. Anim. Behav. Process. 9: 248-265.

Brooks, D.C. and Bouton, M.E. 1993. A retrieval cue for extinction attenuates spontaneous recovery. J. Exp. Psychol. Anim. Behav. Process. 19: 77-89.

Brooks, D.C., Palmatier, M.I., Garcia, E.O., and Johnson, J.L. 1999. An extinction cue reduces spontaneous recovery of a conditioned taste aversion. Anim. Learn. Behav. 27: 77-88.

Davis, M. 2002. Role of NMDA receptors and MAP kinase in the amygdala in extinction of fear: Clinical implications for exposure therapy. Eur. J. Neurosci. 16: 395-398.

Falls, W.A., Miserendino, M.J.D., and Davis, M. 1992. Extinction of fear-potentiated startle: Blockade by infusion of an NMDA antagonist into the amygdala. J. Neurosci. 12: 854-863.

Frohardt, R.J., Guarraci, F.A., and Bouton, M.E. 2000. The effects of neurotoxic hippocampal lesions on two effects of context after fear extinction. Behav. Neurosci. 114: 227-240.

Garcia, R. 2002. Stress, synaptic plasticity, and psychopathology. Rev. Neurosci. 13: 195-208.

Herry, C. and Garcia, R. 2002. Prefrontal cortex long-term potentiation, but not long-term depression, is associated with the maintenance of extinction of learned fear in mice. J. Neurosci. 22: 577-583.

McDonald, A.J., Mascagni, F., and Guo, L. 1996. Projections of the medial and lateral prefrontal cortices to the amygdala: A Phaseolus vulgaris leucoagglutinin study in the rat. Neuroscience 71: 55-75.

Pavlov, I.P. 1927. Conditioned reflexes. Oxford University Press, Oxford.

Quirk, G.J. 2002. Memory for extinction of conditioned fear is long-lasting and persists following spontaneous recovery. Learn. Mem. 9: 402-407.

Rescorla, R.A. 2001. Retraining of extinguished Pavlovian stimuli. J. Exp. Psychol. Anim. Behav. Process. 27: 115-124.

Rosenkranz, J.A. and Grace, A.A. 2002. Cellular mechanisms of infralimbic and prelimbic prefrontal cortical inhibition and dopaminergic modulation of basolateral amygdala neurons in vivo. J. Neurosci. 22: 324-337.

Santini, E., Muller, R.U., and Quirk, G.J. 2001. Consolidation of extinction learning involves transfer from NMDA-independent to NMDA-dependent memory. J. Neurosci. 21: 9009-9017.

Walker, D.L., Ressler, K.J., Lu, K.T., and Davis, M. 2002. Facilitation of conditioned fear extinction by systemic administration or intra-amygdala infusions of D-cycloserine as assessed with fear-potentiated startle in rats. J. Neurosci. 22: 2343-2351.

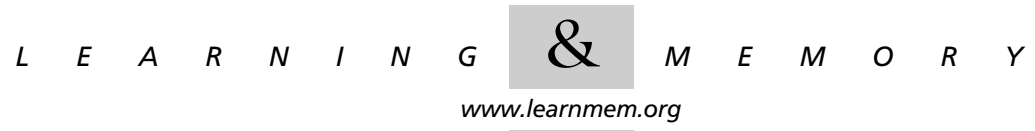




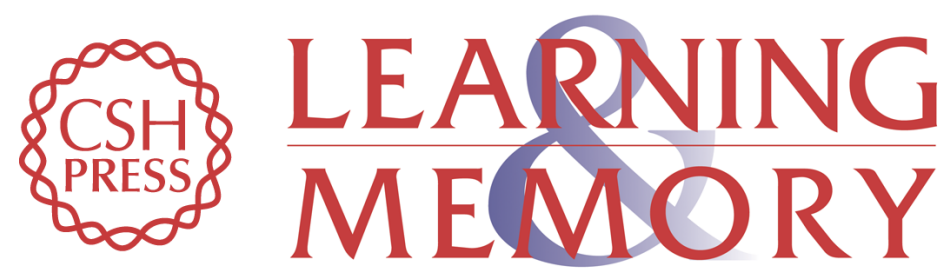

\section{Postextinction of Conditioned Fear: Between Two CS-Related Memories}

René Garcia

Learn. Mem. 2002, 9:

Access the most recent version at doi:10.1101//m.56402

References This article cites 15 articles, 6 of which can be accessed free at:

http://learnmem.cshlp.org/content/9/6/361.full.html\#ref-list-1

License

Email Alerting Receive free email alerts when new articles cite this article - sign up in the box at the top Service right corner of the article or click here. 